and therefore simply using peak velocity might give a less reliable optimum? Surely the time saved by using peak would have a price to pay in poorer reproducibility of the optimum? In this study, we evaluate whether peak velocity is a suitable alternative to VTI, having regard to both time consumed and reproducibility. We also examine whether averaging multiple replicate measurements improves optimisation.

Methods \& Results VV optimisation was performed on 40 subjects with biventricular pacemakers using LVOT velocity (VTI or peak) as the echocardiographic marker being maximised. Importantly, 6 successive replicate optimisations were performed per patient at a single session. Scatter of apparent VV optimum between repeat optimisations was threefold smaller for peak than VTI $(p<0.03)$, with a single measurement for each. Peak velocity had a higher intraclass correlation coefficient (ICC) than VTI ( 0.66 vs 0.53 , $p=0.003$ ). Scatter between replicate optimisations is reduced if, instead of single measurements, we use pairs, or triplicates (ANOVA $p<0.0001)$. This benefit occurs with both peak and VTI ( $p<0.001$ among each). Time taken for acquisition and analysis of a single optimisation (6 settings) was $17.5 \mathrm{~s}$ for peak and $57.5 \mathrm{~s}$ for VTI $(\mathrm{p}<0.0001)$.

Conclusions Doppler optimisation of VV delay using peak velocity rather than VTI is (as expected) quicker but (surprisingly) more accurate. Making replicate measurements further improves reproducibility. Perhaps guidelines should favour peak over VTI and mandate multi-replicate averaging? These data suggest a rare opportunity to reduce labour while increasing reliability of optimisation. Indeed, triplicate peak velocity assessment takes the same amount of time as a single VTI, and identifies the VV optimum 3 times more confidently. While VTI measurement remains essential for assessing stroke volume and cardiac output, for optimisation purposes it comparison of peak velocity between different settings is both faster and more reliable.

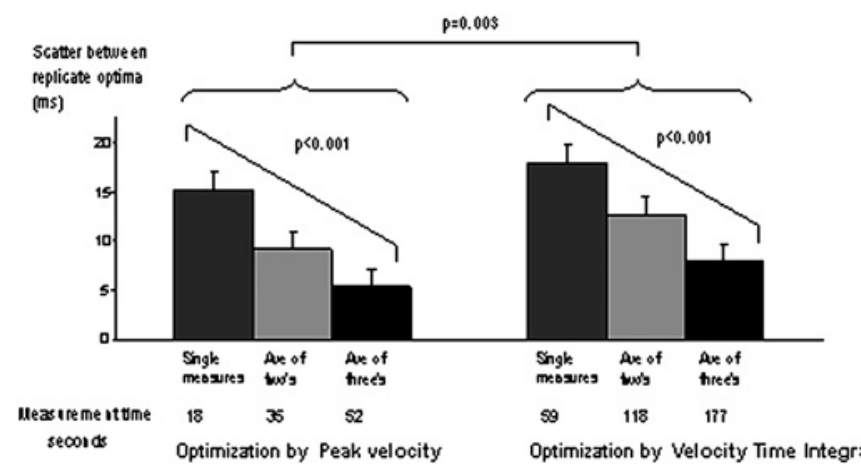

Abstract 87 Figure 1

\section{EVALUATION OF THE IMPACT OF AV DELAY VARIATION ON THE ACUTE MECHANOENERGETIC EFFICIENCY OF CARDIAC RESYNCHRONISATION THERAPY AND ASSESSMENT OF PERFORMANCE OF NON-INVASIVE VS INVASIVE HAEMODYNAMIC OPTIMISATION}

doi:10.1136/heartjnl-2011-300198.88

A Kyriacou, P Pabari, K Willson, R Baruah, S Sayan, D W Davies, J Mayet, N S Peters, P Kanagaratnam, Z Whinnett, D P Francis. International Centre for Circulatory Health, London, UK

Background The impact of varying AV delay on the acute mechanoenergetic efficiency of cardiac resynchronisation therapy (CRT) is not known; nor is known if non-invasive haemodynamic optimisation by blood pressure agrees with invasive haemodynamic measures during optimisation. We studied these invasively, in contemporary patients.

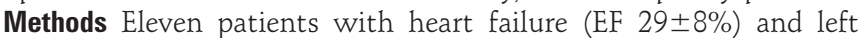
bundle branch block (LBBB, ORS $154 \pm 26 \mathrm{~ms}$ ) underwent measure- ments of left ventricular (LV) pulse pressure (systolic minus diastolic), aortic flow velocity and myocardial oxygen consumption (MVO2) at four settings: $3 \mathrm{AV}$ delays during biventricular (BiV) pacing (reference BiV-AV120 ms; BiV-AV40 ms; individualised haemodynamic BiVAVoptimum), and at intrinsic ventricular conduction (LBBB). Atrial pacing at $100 \mathrm{bpm}$ ensured a fixed heart rate.

Results LV pulse pressure rose from LBBB to BiV-AV120 ms by $10 \pm 2 \%(p<0.001)$ and $2 \pm 1 \%$ more $(p<0.05)$ at the haemodynamic BiV-AVoptimum. At BiV-AV40 ms, pressure was $10 \pm 2 \%$ worse than BiV-AV120 ms $(p<0.001)$, no different to LBBB $(\Delta=0.8 \pm 0.4 \%$, $\mathrm{p}=\mathrm{ns}$ ). Invasive aortic flow velocity, measured at a fixed position throughout each individual's study (ie, cardiac output index), rose by $9 \pm 2 \%(p<0.01)$ from LBBB to BiV-AV120 ms, rising a further $3 \pm 1 \%(p<0.01)$ at BiV-AVoptimum. At BiV-AV40 ms, aortic flow was, no different to LBBB ( $p=N S$ ). MVO2 increased from LBBB to BiV-AV120 ms by $9 \pm 4 \% \quad(p=0.035)$ and to BiV-AVoptimum by $12 \pm 3 \%(p=0.002) . M V O 2$ at At BiV-AV40 ms and LBBB was not significantly different $(\Delta 4 \pm 3 \%, \mathrm{p}=\mathrm{ns})$, The 4 pacing states lay on a straight line: for $\Delta$ pressure against $\Delta$ flow, $r=0.99(p<0.01)$, Abstract 88 figure 1. $\Delta$ external work $(\Delta$ pressure $\times \Delta$ flow) correlated with $\Delta$ MVO2, $\mathrm{r}=0.99(\mathrm{p}<0.01)$, with slope $1.61 \pm 0.17$, significantly greater than $1.00(\mathrm{p}<0.05)$, Abstract 88 figure 2 .

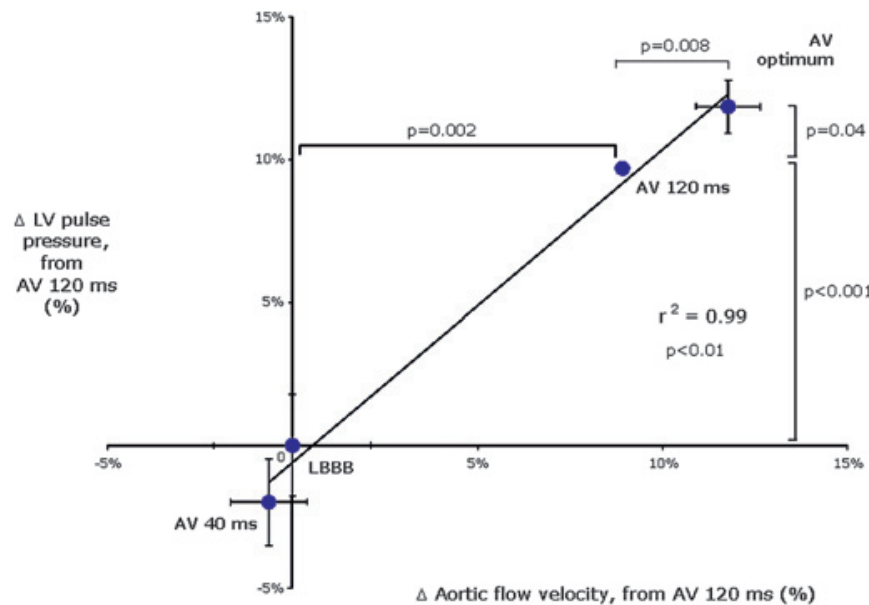

Abstract 88 Figure 1 The correlation of LV pulse pressure and aortic flow velocity during acute biventricular pacing, (at three AV delays) and during LBBB, at a fixed heart rate.

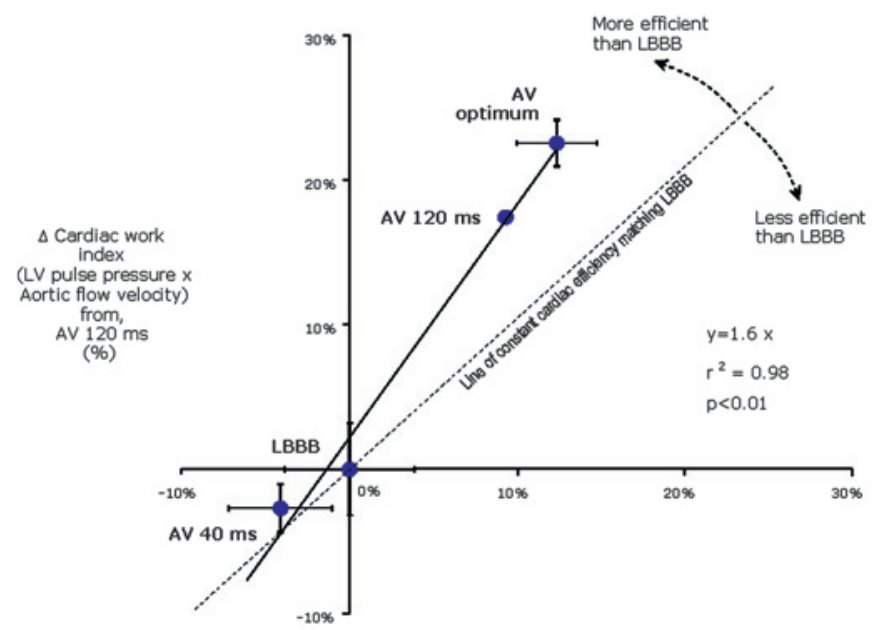

$\Delta$ Myocardial oxygen consumption, from AV $120 \mathrm{~ms}(\%)$

Abstract 88 Figure 2 The correlation of cardiac work and myocardial oxygen consumption during acute biventricular pacing, (at three AV delays) and during LBBB, at a fixed heart rate. 
The correlations of optimal AV delays by non-invasive (Finometer) systolic blood pressure (SBP) vs invasive measures were as follows; aortic SBP, $\mathrm{r}^{2}=0.96, \mathrm{p}<0.01$; aortic flow velocity, $\mathrm{r}^{2}=0.81$, $\mathrm{p}<0.01 ; \mathrm{LV} \mathrm{dP} / \mathrm{dtmax}, \mathrm{r}^{2}=0.68, \mathrm{p}<0.01$.

Conclusions During acute biventricular pacing, at a fixed heart rate, changing the AV delay affects the cardiac mechanoenergetics. When an AV delay improves external cardiac work, compared to LBBB or a physiologically too short AV delay (eg, AV $40 \mathrm{~ms}$ ), it also increases the myocardial oxygen consumption. However, only $1 \%$ more energy is consumed per $1.6 \%$ more external work (pressure $\times$ flow) done; as a result cardiac efficiency improves. Haemodynamic optimisation of AV delay can be achieved with high precision using non invasive beat-to-beat pressure measurements. This should enable routine haemodynamic optimisation (easily automated) of CRT devices in clinical practice.

\section{ELECTROMECHANICAL INTERACTION IN PATIENTS UNDERGOING CARDIAC RESYNCHRONISATION THERAPY: COMPARISON OF INTRACARDIAC ACTIVATION MAPS AND EARLY SEPTAL CONTRACTION IN LEFT BUNDLE BRANCH BLOCK}

doi:10.1136/heartjnl-2011-300198.89

${ }^{1} \mathrm{~S}$ G Duckett, ${ }^{2} \mathrm{O}$ Camara, ${ }^{1} \mathrm{M}$ Ginks, ${ }^{3} \mathrm{~J}$ Bostock, ${ }^{1} \mathrm{P}$ Chinchapatnam, ${ }^{1} \mathrm{M}$ Sermesant, ${ }^{2} \mathrm{~A}$ Pashaei, ${ }^{3} \mathrm{~J} S \mathrm{~S}$ Gill, ${ }^{3} \mathrm{G}$ Carr-White, ${ }^{2} \mathrm{~A} F$ Frangi, ${ }^{1} \mathrm{R} S$ Razavi, ${ }^{2} \mathrm{~B} H$ Bijnens, ${ }^{3} \mathrm{C}$ A Rinaldi. ${ }^{1}$ Kings College London, London, UK; ${ }^{2}$ UPF, Barcelona, Spain; ${ }^{3} G u y$ 's and St Thomas' Hospital, London, UK

Introduction Early inward motion and thickening/thinning of the ventricular septum associated with left bundle branch block (LBBB) is known as the septal flash (SF). Correction of SF corresponds with CRT response. We hypothesise that electromechanical interactions induced by SF are associated with functional changes in conductivity and a "U-shaped" activation pattern. Characterising the spatio-temporal relationship between electrical and mechanical events may explain why patients with a SF respond to CRT. Methods 13 patients (63 \pm 10 years, 10 men) with severe heart failure (EF 22.8 $\pm 5.8 \%$ ) undergoing CRT underwent echocardiography and non-contact mapping (NCM) pre-implant. Presence and extent of a SF was defined visually and with $\mathrm{M}$-mode and fused with NCM bull's eye plots of endocardial activation patterns. LV $\mathrm{dP} / \mathrm{dtmax}$ was measured during different pacing modes.

Results Five patients had a large SF, four small SF and four no SF. Patients with large SF had areas of conduction block in noninfarcted regions whereas those with small or no SF did not (Abstract 89 figure 1). Patients with large SF had greater acute response to left ventricular (LV) and biventricular (BIV) pacing vs those with small/no SF (\% increase $\mathrm{dP} / \mathrm{dt} 28 \pm 14 \%$ vs $11 \pm 19 \%$ for LV pacing and $42 \pm 28 \%$ vs $22 \pm 21 \%$ for BIV pacing). The lines of conduction block disappeared after LV and BIV pacing, while remaining present with RV pacing (Abstract 89 figure 2). Abstract 89 figure 1 Patient with a large SF. Unipolar isochronal map with NCM electrograms showing fragmented signals (development of split potentials) indicating a reduction of conduction and inability to cross throughout the inferior region. The NCM mapping electrograms show the criteria used by Auricchio et al to define block, with the emergence of R-wave, smallest and earliest at the superior part of the block (where area of block begins) with largest negative peak. Bold white arrows on the electrogram indicate how the electrical activation spreads superiorly in a U-shape pattern leading to the development of split potentials. Abstract 89 figure 2 Activation maps of patient with a large SF. Row A, baseline with area of block and late anterior breakthrough. Row B, RV pacing showing the area of anterior block remains. Row C, BIV pacing. Functional conduction block has disappeared.

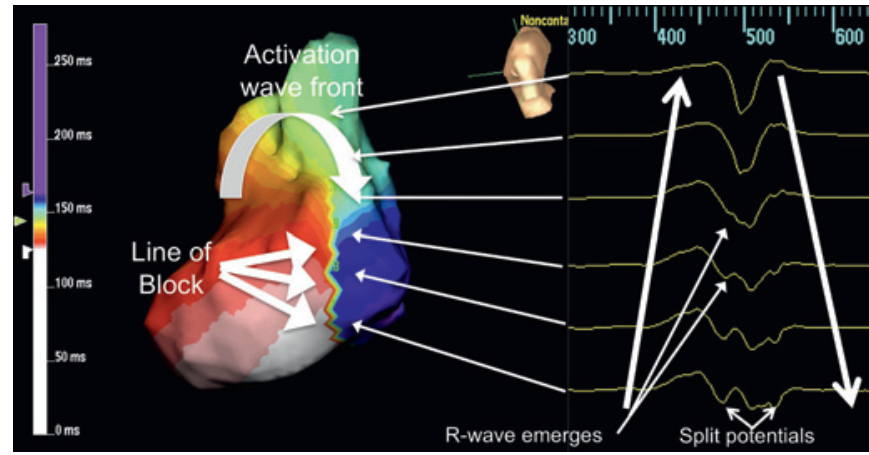

Abstract 89 Figure 1

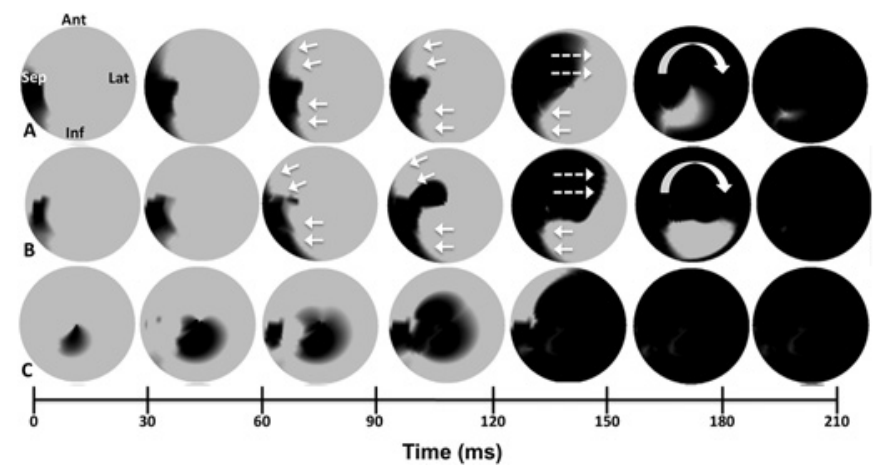

Abstract 89 Figure 2

Conclusion A strong interaction exists between electrical activation and mechanical deformation of the septum. Correction of both mechanical synchrony and the functional conduction block by CRT may explain the large positive response in patients with a SF.

\section{INVASIVE ACUTE HAEMODYNAMIC RESPONSE TO GUIDE LV LEAD IMPLANTATION PREDICTS CHRONIC REMODELLING IN PATIENTS UNDERGOING CARDIAC RESYNCHRONISATION THERAPY}

doi:10.1136/heartjnl-2011-300198.90

${ }^{1} \mathrm{~S}$ G Duckett, ${ }^{1} \mathrm{M}$ Ginks, ${ }^{1} \mathrm{~A}$ Shetty, ${ }^{2} \mathrm{~J}$ Bostock, ${ }^{2} \mathrm{~J} S \mathrm{~S}$ ill, ${ }^{2} \mathrm{~S}$ G Hamid, ${ }^{2} \mathrm{~S}$ Kapetanakis, ${ }^{2} \mathrm{E}$ Cunliffe, ${ }^{1} \mathrm{R} S$ Razavi, ${ }^{2} \mathrm{G}$ Carr-White, ${ }^{2} \mathrm{C}$ A Rinaldi. ${ }^{1}$ Kings College London, London, UK; ${ }^{2}$ Guy's and St Thomas' Hospital, London, UK

Introduction Cardiac resynchronisation therapy (CRT) reduces mortality and morbidity in heart failure patients, however up to 30\% of patients do not derive symptomatic benefit. Higher proportions do not remodel. Multi-centre trials have shown echocardiographic techniques are poor at improving response rates. We hypothesised that the degree of acute haemodynamic response (AHR) at implant can predict which patients remodel. We evaluated the relationship between AHR and reverse remodelling (RR) in CRT. Methods 33 patients undergoing CRT (21 dilated \& 12 ischaemic cardiomyopathy) were studied. Left ventricular (LV) volumes were assessed pre and post CRT. AHR (LV-dP/dtmax) was assessed at implant using a pressure wire in the LV cavity. The LV lead was placed in potential target veins and the largest percentage rise in $\mathrm{LV}-\mathrm{dP} / \mathrm{dtmax}$ from baseline (AAI or RV pacing with atrial fibrillation) to DDDLV was used to determine optimal LV lead position. RR was defined as reduction in LV end systolic volume (ESV) $\geq 15 \%$ at 6 months.

Results LV-dP/dtmax increased significantly from baseline $(801 \pm 194 \mathrm{~mm} \mathrm{Hg} / \mathrm{s}$ to $924 \pm 203 \mathrm{~mm} \mathrm{Hg} / \mathrm{s} \quad(\mathrm{p}<0.001))$ with DDDLV pacing for the optimal LV lead position. There was a significant difference in the percentage rise in LV-dP/dtmax between the best and worst LV lead position (Abstract 90 figure 1). LVESV 